\section{MAKING EVERY DAY COUNT FOR PATIENTS IN AN ACUTE HOSPITAL AT THE END OF LIFE: SILVER TO GOLD BED DAYS}

C Hayle, E Given, A Hayes, B Houghton, R Latten, M Maxwell, A Pattullo, G Purewal, S Shouls, C Sanders. Wirral University Teaching Hospital Foundation Trust, Wirral Community Foundation Trust, Hospice UK/National Council for Palliative Care, NHS Improvement

\subsection{6/bmispcare-2018-ASPabstracts.167}

Background Wirral University Teaching Hospitals NHS FT serves a population of 330,000 . Patients who subsequently die in hospital spend 21 days (mean) of their last 100 days of life in hospital. When time is so precious, it is crucial that every day spent in hospital 'counts'.

Inpatient specialist palliative care and end of life care provisions was fragmented. The aim was to improve care and cohesiveness by putting patient's wishes at the centre of clinical process.

Methods The 'red to green days' concept (developed for Safer Patient Flow) was adapted to focus on patient experience of care. At the start of the day a board round assesses 'what matters most today' for each patient, with agreed actions across the team. The patient's day starts as silver and is then recorded as 'gold' only if progress is made against the patient's expressed wishes. A silver day means a patient receives high quality care, but no action to achieve wishes. The team applied 'Plan Do Study Act' methods to develop this approach from concept to practice.

Results Patients' wishes were more consistently understood and met. Examples include: obtaining a wig, a urinary catheter, complex spiritual care, wedding ceremony, rapid discharge, hospice bed, physical symptom control. The focus on 'what matters most' to patients increased team cohesiveness, greater peer support and shared responsibility rather than individual caseloads.

Performance was monitored using Statistical Process Control and ongoing analysis of barriers to achieving a gold day. There is an association between patient flow and the ability of the SPC team to meet patient wishes.

Conclusions A singe focus on patients' wishes and what matters to them helps team working together to meeting these goals. Collecting evidence on why patients are unable to achieve their wishes supports thematic learning to guide future commissioning and service developments.

\section{DATA SHARING PROJECTS IN END OF LIFE CARE - EASIER, HARDER, OR JUST AS FIERCELY COMPLEX AS ANY OTHER?}

Mila Petrova, Stephen Barclay. University of Cambridge

10.1136/bmjspcare-2018-ASPabstracts. 168

Background Electronic Palliative Care Coordination Systems (EPaCCS), enabling data sharing across care settings, have been under development in England for over nine years and are expected to cover all areas of the country by 2020. Data sharing (technically termed 'health information exchange'?, HIE) projects are, however, amongst the most difficult projects of health information technology (HIT) implementation. We report on a sub-study of 'Prepared to Share?', a mixed methods realist evaluation of The Cambridgeshire and Peterborough
Project for Data Sharing in End of Life Care, nested in broader research on data sharing and EPaCCS.

Methods 44 participants were interviewed using principles of realist and episodic interviewing. Preliminary analysis of nine interviews and four existing reviews on HIE and HIT, covering 135 studies, contributed to an initial analysis framework. Framework and thematic analysis principles and 'pluralist' coding informed by the philosophy of science of J. Dupre were applied in the analysis.

Results We identified $>460$ challenges and $>300$ drivers to implementing EPaCCS and data sharing projects more broadly, the majority of them richly described in the interviews. The main sources of challenges were: the existence of 'alternatives', i.e. primarily well entrenched and thus easier ways of working, but also other data sharing solutions; generic resource constraints within the health system; information governance issues; generic IT infrastructure challenges; insufficient adaptability, capacities for personalisation and flexibility of the solution; and generic features of the health system, such as its fragmentation. 'Expected benefits' and 'training, education and awareness raising' were the only higher-level categories where drivers outweighed challenges.

Conclusions EPaCCS projects and research need to draw on and contribute more to existing HIE and HIT research. EPaCCS teams and users are learning the hard way many lessons which are already described in this broader research literature.

\section{TRIGGERS FOR REFERRAL TO PALLIATIVE CARE}

R Gemmell, K Mackay, N Yousaf, J Droney. Royal Marsden Hopsital

\subsection{6/bmjspcare-2018-ASPabstracts. 169}

Background Benefits of early palliative care (PC) referral for oncology patients are well-documented. The development of PC referral 'Triggers' may help identify which patients should be referred. A number of 'Triggers' have been proposed, but are currently not commonly used in clinical practice. This project aimed to evaluate a number of published referral Triggers through a retrospective note review of PC team involvement with patients who died during a non-elective hospital admission.

Method All patients admitted to the oncology hospital nonelectively during one year who died during that admission were selected, excluding patients admitted to CCU $(\mathrm{N}=159)$. The timing of referral to PC, and whether patients met any published PC referral triggers before and during their terminal admission was assessed.

Results 88\% (140/159) of patients would have been eligible to 'trigger' a PC referral during their terminal admission, no matter which Triggers tool was used. Information relating to disease and symptom-related 'Triggers' are most commonly documented in medical notes. Psychosocial factors were less well-documented.

$46.5 \%$ of patients were known to PC prior to their final admission. All but 3 of the remaining patients were referred during admission, with a median time (range) between admission and referral of 4 days (0-58 days), and between referral and death of 9 days (0-90 days).

Conclusions Currently, many patients are not known to palliative care until their last hospital admission, and therefore until their last days of life. In this patient cohort, a palliative 